\title{
A Computational Parcellated Brain Model for Electric Field Analysis in Transcranial Direct Current Stimulation
}

\author{
M. A. Callejón-Leblic and Pedro C. Miranda
}

\section{Introduction}

tDCS is a noninvasive brain modulation technique which produces cortical excitatory and inhibitory plastic changes by delivering small-amplitude electric currents into the brain through two or more electrodes placed on the scalp [1]. Although neuromodulation has shown promising results regarding the improvement of both cognitive and motor functions as well as the treatment of several neurological and neuropsychiatric diseases [2-4], the field still faces one major drawback: the lack of reproducibility caused by large differences among subjects and studies [5].

Realistic computational models derived from magnetic resonance imaging (MRI) have proved useful for the estimation of the EF magnitude and distribution through the brain, revealing the effect of individual anatomy as a major cause for intersubject variability [6-10]. Further to this, classic electrode placement criteria, where one electrode is usually located above the neural target and another some distance away, has been called into question when predicting similar or even higher values in both superficial and deep areas between the electrodes [10-14]. It is for this reason that the correlation between the EF magnitude and the observed neurophysiological effects of tDCS continues to be controversial [15-17]. Recent modelling studies have emphasized that the influence of tDCS-induced EFs does not only depend on

\footnotetext{
M. A. Callejón-Leblic

Biomedical Engineering Group, University of Seville, Seville, Spain

Instituto de Biofísica e Engenharia Biomédica, Faculdade de Ciências, Universidade de Lisboa, Lisboa, Portugal

e-mail: mcallejon@us.es

P. C. Miranda $(\square)$

Instituto de Biofísica e Engenharia Biomédica, Faculdade de Ciências, Universidade de Lisboa, Lisboa, Portugal

e-mail: pcmiranda@fc.ul.pt
} 
their magnitude but also on their relative orientation to specific cortical targets [1820].

This chapter is structured as followed: Section 2 offers a brief review of recent works addressing the intricate relationship between the modelled EF magnitude and/or orientation and the stimulation response. Based on previous work in [21], Sect. 3 describes the modelling framework followed to obtain a computational parcellated brain model based on the finite element method (FEM) for the analysis of the distribution of tangential and normal EF components over the cortex. Section 4 presents estimates of mean and peak tangential and normal EF values for different cortical regions and four different electrode montages typically used in tDCS clinical applications. Section 5 discusses the main differences observed between the various electrode montages analyzed. Finally, Sect. 6 concludes this chapter.

\section{Relation Between EF Magnitude and Orientation and tDCS-Physiological Effects}

The conventional criteria for the placement of electrodes in tDCS stems from initial studies undertaken by Nitsche et al. where significant modulation of motor-evoked potentials (TMS-MEPs) was found only for the left M1-right supraorbital area (RSOA) montage [22, 23]. In apparent contradiction to this, current MRI-driven computational EF models predict considerable EF spread over intermediate and deep brain regions between the electrodes, relatively far from the presumed targeted regions [9, 11, 14, 24, 25].

As demonstrated through animal and in vitro studies, changes in membrane excitability of neurons are sensitive to orientation of EFs relative to different neuronal compartments [26-28]. However, given the complex anatomy of the highly convoluted human cortex, extrapolating these results to in-vivo studies in humans is not straightforward. Figure 1 shows an illustrative scheme of the EF vector over the human cortical sheet and the definition of both tangential and normal EF components. Recent multiscale models integrating both macroscopic and microscopic effects at both tissue and cellular levels have revealed a high correlation between soma polarization and normal EF irrespective of electrode montage [29].

At a population level, recent works have investigated the correlation between the simulated EF magnitude and/or orientation and the tDCS-induced excitatory effects, with seemingly divergent results. For instance, as reported by Fitscher et al. in [30], lower magnitude and normal EF values estimated for multifocal tDCS of the motor cortex did not explain the increased excitability changes observed when compared with standard left M1-RSOA montage. More recently in [31], Antonenko et al. applied tDCS to 24 healthy participants during eyes-closed resting-state functional resonance imaging for the standard large-pad left M1-RSOA montage under anodal, cathodal, and sham stimulation conditions. A better correlation with physiological effects was identified for the EF magnitude rather than for the normal component 


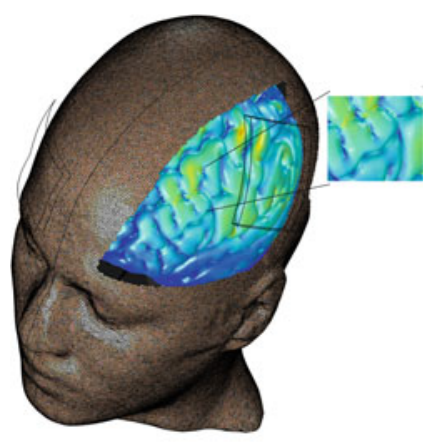

A

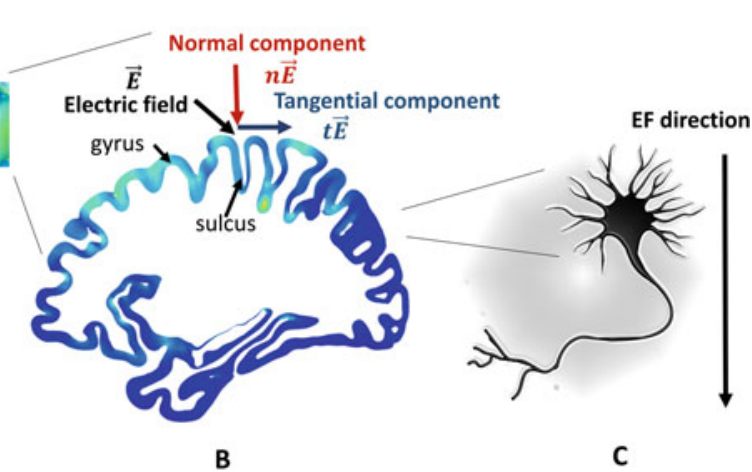

Fig. 1 Schematic illustration of the electric field and their directional components. (a) Realistic computational EF model at tissue level. (b) Description of tangential and normal EF components over a slice of the cortical sheet. (c) Relation between the orientation of EF and the morphology of neuronal structures at cellular level

over the targeted left precentral gyrus for both anodal and cathodal conditions. In another recent work, Foerster et al. [32] analyzed the TMS-MEP excitability response in 15 volunteers after $15 \mathrm{~min}, 1 \mathrm{~mA}$ anodal and cathodal stimulation with two different orientations of the $5 \mathrm{~cm} \times 7 \mathrm{~cm}$ electrode placed over the left M1: one with the long axis of the electrode aligned with the medial-lateral direction and another with the electrode rotated $45^{\circ}$ clockwise. The second electrode was fixed over the contralateral supraorbital area. Although modelling results in a representative brain model did not reveal significant differences regarding EF orientation over M1 for these two montages, a significant enhancement of the excitatory response for both anodal and cathodal conditions was only exhibited with the $45^{\circ}$-rotated electrode. This outcome was instead related to higher EF magnitude over M1 and premotor areas.

Nevertheless, other authors have recently uncovered evidence that the tDCS excitatory changes are indeed sensitive to EF orientation relative to the cortical surface. For example, in [20], Laakso et al. measured the MEPs in 28 healthy subjects before and after a 20 min sham or $1 \mathrm{~mA}$ anodal tDCS stimulation with large-pad electrodes over the right motor cortex. Opposite effects in modulated MEPs were observed for individuals with the strongest and weakest EFs, respectively, showing a high correlation with the normal component in the hand knob area near the TMS hotspot. In another study [18], Rawji et al. compared the tDCSinduced changes measured by TMS-MEP in 22 healthy volunteers by comparing two different montages with two small electrodes placed $7 \mathrm{~cm}$ around M1: one montage with the electrodes aligned so as to direct current perpendicularly to the central sulcus in the posterior-anterior (PA) direction and another with the electrodes directing current parallel to the central sulcus in the medial lateral (ML) direction. Significant after-effects of TMS-MEP for the PA montage were correlated with predicted current density normally oriented toward the hand region, whereas the inexistence of excitatory effects for the ML montage were related to a non-uniform 
EF orientation in this area. Likewise in [19], Hannah et al. confirmed and furthered these results for behavioral motor learning and two PA- and ML-orientated montages over the sensorimotor cortex.

In this section, we have summarized the main results derived from recent works addressing the relationship between the modelled EFs and the tDCS neurophysiological effects. It has been seen that a consensus on EF magnitude or orientationbased mechanisms has not yet been reached. The variability observed among experimental trials may be due to different stimulation parameters and electrode montages used as well as to different modelling approaches. Nonetheless, we can conclude that computational EF models were vital to improve our understanding of the complex relationship between simulated EF magnitude and orientation and the stimulation phenomena observed in tDCS experiments. In this chapter, a computational analysis of the distribution of tangential and normal EF components over a representative brain model is presented for various common tDCS clinical montages.

\section{A Computational Parcellated Brain Model in tDCS}

\subsection{Head Anatomy}

It is common practice for the creation of a realistic computational model of the head to involve the segmentation of T1- and T2-weigthed magnetic resonance images (MRI) based on individual anatomies. The process of converting MRI to EF distribution is further explained in [33]. That said, computational models based on brain atlases have also gained interest among researchers due to their ability to represent an average human brain. Such is the case of the computational model this chapter will focus on, which is based on the $0.5 \mathrm{~mm}^{3}$ ICBM152 V2009b symmetric template derived from the nonlinear average of MRI scans of 152 adults with high anatomical detail of inner brain tissues [34]. Here, an earlier version of SimNIBS [35] (v2.0, http://simnibs.de/) which employs Freesurfer (https://surfer.nmr.mgh. harvard.edu/), and is reported to provide higher resolution of cortical gyri and sulci morphology [36], was used to segment and to obtain 3D surface meshes of ICBM152 inner brain tissues such as gray matter (GM), white matter (WM), cerebellum, and ventricles. Regarding non-brain tissues, Huang et al. in [37] co-registered and re-sliced a prior version of the same template, ICBM152 v6, which better retained the anatomical characteristics of the scalp and the skull, to the same MRI space of ICBM152 V2009b. By artificially overlapping the resultant MRI with an average of several heads, they were able to extend the field of view (FOV) down to the neck and thus generate a full head model named ICBM-NY (New-York) head model. The ICBM-NY segmentation masks made available by Huang et al. were tessellated here to obtain the 3D triangular surface meshes for the outer head tissues such as the scalp, skull, eyeballs, air cavities, and CSF. A non-manifold assembly of these tissues as well as post-processing operations such as the smoothing, re-meshing, and cleaning of small anatomical skull details and 

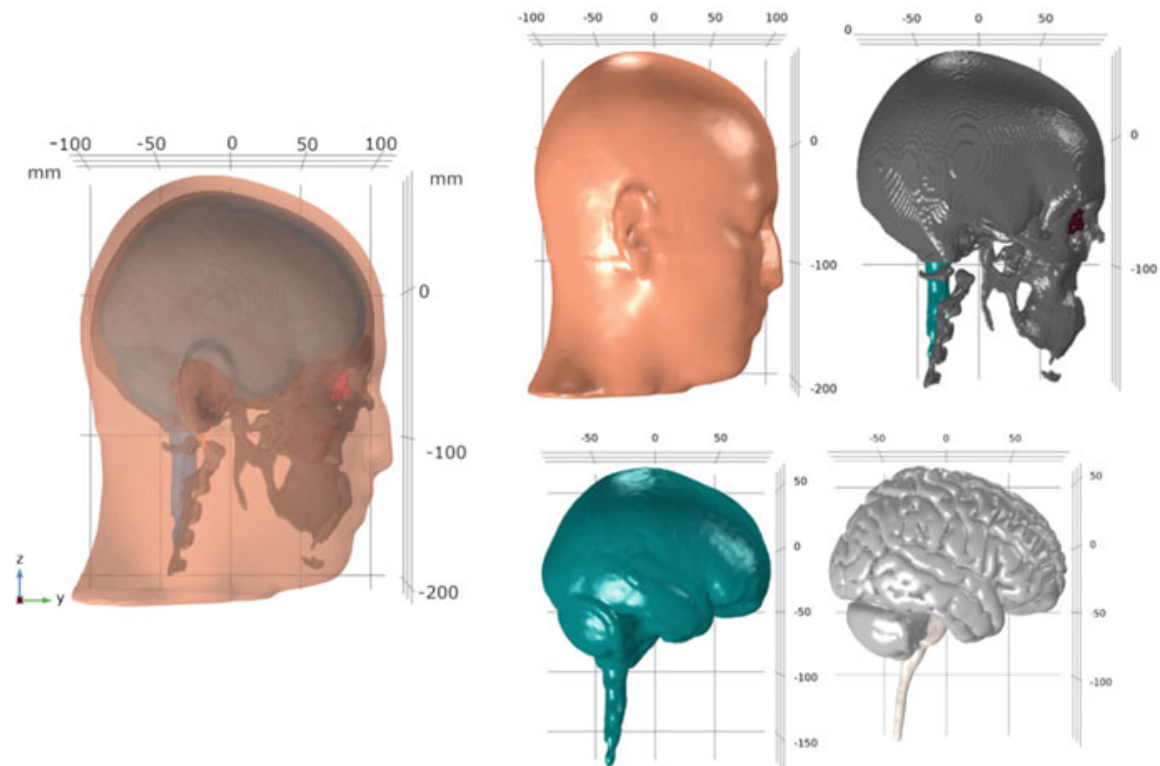

Fig. 2 View of the computational head model based on ICBM152 V2009b template and ICBM152-NY segmentations masks provided by Huang et al. in [37]. The model comprised different tissues such as the scalp, skull, vertebrae, eyeballs, air cavities, CSF, GM, WM, cerebellum, brain stem, and ventricles. Skull openings such as the eye foramina and foramen magnum were manually modelled using Mimics 3-matic

other tissue irregularities were manually carried out in Mimics 3-matic software (v16, https://www.materialise.com/es/medical/software/mimics). The resulting computational head model based on ICBM152 template can be seen in Fig. 2.

\subsection{Cortex Parcellation}

The use of multimodal neuroimaging studies along with EF modelling may help identify the true targeted cortical regions in tDCS. Hence, the delineation of different cortical or brain regions, often referred to as brain parcellation [38], may help provide useful quantitative comparison of the EF dose delivered to different brain substructures. Many neuroimaging and EF modelling software toolboxes include specific labelling tools for brain parcellation based on available brain atlases. The multimodal parcellation reported in [39], considered one of the most detailed in the literature so far, is that derived from the Human Connectome Project version 1.0 (HCP-MMP 1.0, https://balsa.wustl.edu/WN56). It offers a description of 180 areas per hemisphere grouped into 22 regions according to cortical anatomy, function, and connectivity criteria. In this work, we labelled these 22 regions in the ICBM152 model (see Fig. 3) based on the Freesurfer version of HCP-MMP 1.0 parcellation 


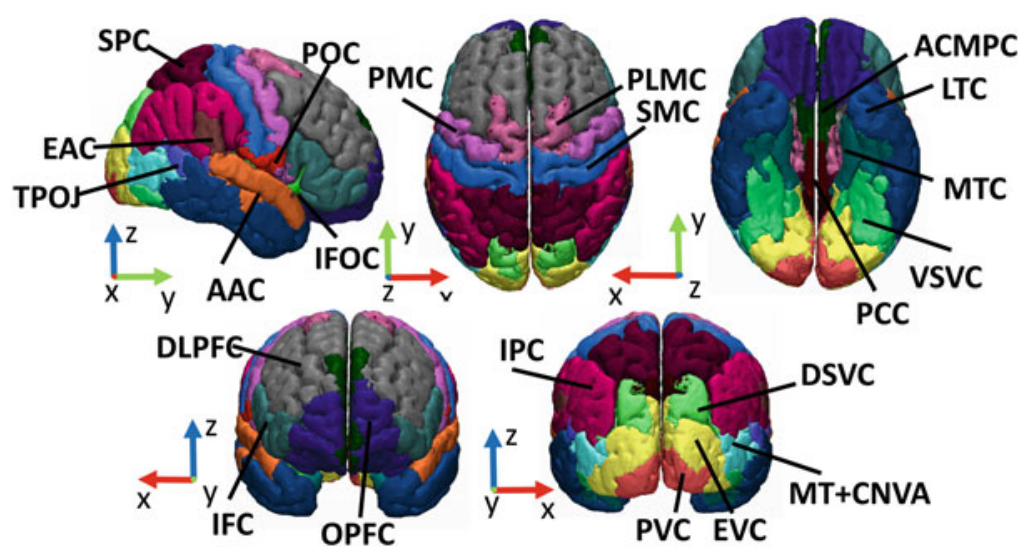

Fig. 3 Cortex parcellated into 22 cortical regions per hemisphere according to color code in Table 1

data [40]. The names of the 22 regions are listed in Table 1, where they have been grouped into 6 main sections per hemisphere, including visual cortex (VC), somatosensory and motor cortex (SMC), auditory cortex (AC), temporal cortex (TC), parietal cortex (PC), and frontal cortex (FC).

\section{3 tDCS Electrode Montages}

Bipolar large-pad saline-soaked electrodes are the most commonly used in tDCS clinical trials. Both anodal (excitatory) and cathodal (inhibitory) stimulations are possible, where the only difference is the direction of the resultant EF but not the distribution [41]. As can be seen in Fig. 4, four bipolar montages consisting of $7 \mathrm{~cm} \times 5 \mathrm{~cm}$ rectangular, 6-mm-thick electrodes were considered. This permitted the analysis of the EF distribution over various cortical areas commonly targeted in tDCS clinical applications: motor cortex (C3-right supraorbital area RSOA), dorsolateral prefrontal cortex (F3-RSOA), visual cortex $(\mathrm{Oz}-\mathrm{Cz})$, and auditory cortex (T8-T7). The scalp coordinates for these positions were identified according to 10-20 EEG system.

\subsection{The Physics of tDCS}

The physics of tDCS is that of a conducting body volume with two or more electrodes attached to its surface [42]. The electric current injected through the attached electrodes causes a net flow of ions through the head tissues, which can be described in terms of an electric field, $\vec{E}(\mathrm{~V} / \mathrm{m})$, or a current density, $\vec{J}=\sigma \vec{E}$ 
Table 1 List of 22 parcellated cortical regions from HCP-MMP 1.0

\begin{tabular}{|c|c|}
\hline & Parcelled Cortical Regions \\
\hline \multirow{5}{*}{$\begin{array}{l}\text { Visual/Occipital } \\
\text { cortex }\end{array}$} & 1. Primary Visual Cortex: PVC \\
\hline & 2. Early Visual Cortex: EVC \\
\hline & 3. Dorsal Stream Visual Cortex: DSVC \\
\hline & 4. Ventral Stream Visual Cortex: VSVC \\
\hline & 5.M.T.+Complex Neighboring Visual Areas: MT+CNVA \\
\hline \multirow{4}{*}{$\begin{array}{l}\text { Somatosensory and } \\
\text { Motor Cortex }\end{array}$} & 6. Somatosensory and Motor Cortex: SMC \\
\hline & 7. Paracentral Lobular and Midcingulate Cortex: PLMC \\
\hline & 8. Pre-Motor Cortex: PMC \\
\hline & 9. Posterior Opercular Cortex: POC \\
\hline \multirow{3}{*}{ Auditory Cortex } & 10. Early Auditory Cortex: EAC \\
\hline & 11. Auditory Association Cortex: AAC \\
\hline & 12. Insular and Frontal Opercular Cortex: IFOC \\
\hline \multirow{2}{*}{ Temporal Cortex } & 13. Medial Temporal Cortex: MTC \\
\hline & 14. Lateral Temporal Cortex: LTC \\
\hline \multirow{4}{*}{ Parietal Cortex } & 15. Temporal-Parietal-Occipital Junction: TPOJ \\
\hline & 16. Superior Parietal cortex: SPC \\
\hline & 17. Inferior Parietal Cortex: IPC \\
\hline & 18. Posterior Cingulate Cortex: PCC \\
\hline \multirow{3}{*}{ Frontal Cortex } & $\begin{array}{l}\text { 19. Anterior Cingulate and Medial Prefrontal Cortex: ACMPC } \\
\text { 20. Orbital and Polar Frontal Cortex: OPFC }\end{array}$ \\
\hline & 21. Inferior Frontal Cortex: IFC \\
\hline & 22. Dorsolateral Prefrontal Cortex: DLPC \\
\hline
\end{tabular}

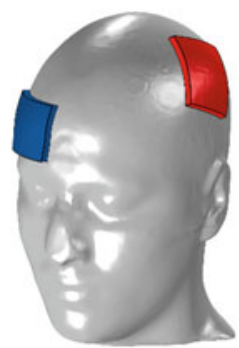

C3-RSOA

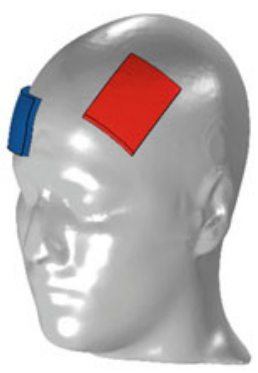

F3-RSOA

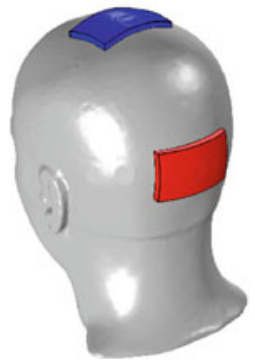

$\mathrm{Oz}-\mathrm{Cz}$

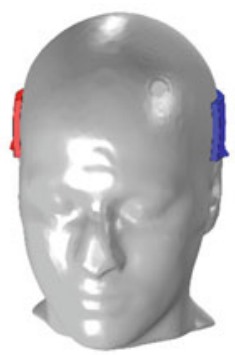

T8-T7

Fig. 4 Electrode montages commonly used in tDCS clinical applications: C3-RSOA, F3-RSOA, $\mathrm{Oz}-\mathrm{Cz}$, and T8-T7. The anode (positive) is colored red and the cathode (negative) blue

$\left(\mathrm{A} / \mathrm{m}^{2}\right)$. In turn, these currents lead to changes in the membrane potential of excitable neurons, which are excited or inhibited depending on the stimulation applied (anodal or cathodal), the magnitude of the electric field induced, and its relative direction to the targeted neural segments. 
This bioelectric problem can be mathematically modelled in terms of Laplace's equation, i.e., $\nabla \cdot(\sigma \nabla \phi)=0$, which provides a stationary solution for the scalar potential, $\Phi$, in the conducting head tissues. From this, the electric field $\vec{E}=\overrightarrow{-\nabla \Phi}$ and the current density, $\vec{J}=\sigma \vec{E}$, can be derived. The required boundary conditions are usually (i) the continuity of the normal current density at internal boundaries, $-\vec{n} \cdot\left(\overrightarrow{J_{1}}-\overrightarrow{J_{2}}\right)=0$; (ii) electrical isolation at external boundaries, $-\vec{n} \cdot \vec{J}=0$; and (iii) floating potential boundary conditions for the injection of a constant electric current $I_{0}$ through the electrodes: in this way, a constant voltage $V=V_{0}$ is applied on the electrode boundary such that the total normal electric current density $\vec{J}$ equals a specific current $I_{0}$, i.e., $\int_{\partial \Omega}(-\vec{n} \cdot \vec{J}) d S=I_{0}$.

\subsection{FEM Calculation}

Given the highly complex anatomy of the human head and brain tissues, an approximate solution of Laplace's equation can only be obtained through the use of numerical computational techniques such as the finite element method (FEM) [43]. Therefore, FEM software such as that used here, Comsol Multiphysics (v5.3a, www.comsol.com), provides a solution for the electric potential $\Phi$ at each node of a tetrahedral volume mesh resembling the head anatomy (see Fig. 5). Another key element of FEM calculation is the electric conductivity of head tissues, $\sigma(\mathrm{S} / \mathrm{m})$, often emulated as homogeneous isotropic conductor volumes. Despite the existing

Fig. 5 Volume mesh for FEM calculation

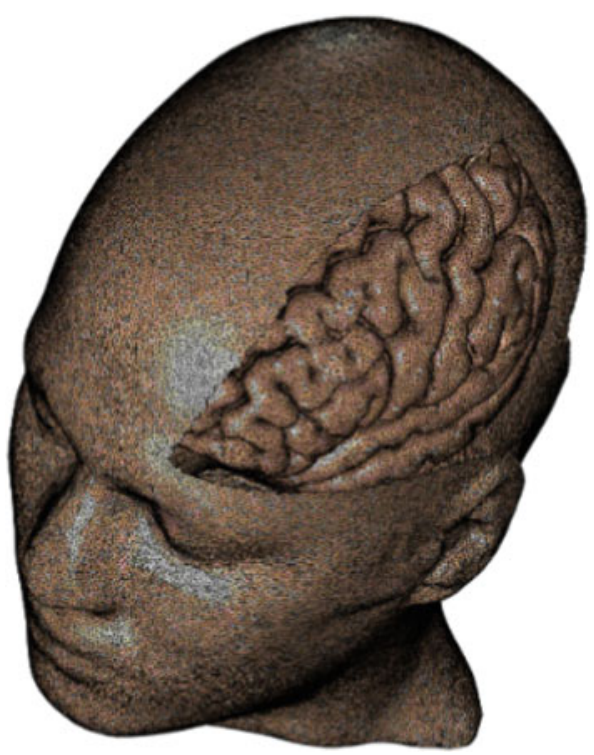


Table 2 Conductivity values in $\mathrm{S} / \mathrm{m}$ for different head tissues, taken from the literature

\begin{tabular}{l|l}
\hline Tissue & Conductivity value $\sigma(\mathrm{S} / \mathrm{m})$ \\
\hline Scalp & 0.33 \\
\hline Skull & 0.008 \\
\hline CSF & 1.79 \\
\hline GM & 0.33 \\
\hline WM & 0.15 \\
\hline Eyeballs & 1.5 \\
\hline Air cavities & $10 \mathrm{e}^{-14}$ \\
\hline Sponge electrodes & 2 \\
\hline
\end{tabular}

controversy on accurate conductivity values, recent in vivo measurements [44] reveal a reasonable agreement with values commonly used in the modelling literature, especially for GM, WM, and scalp, as shown in Table 2 [41].

\section{Results}

\subsection{Tangential and Normal EF Distribution Through the Cortex}

Tangential $|t \vec{E}|$ and normal $|n \vec{E}| \mathrm{EF}$ components calculated over the cortical surface are shown in Figs. 6 and 7 for an injected current of $1 \mathrm{~mA}$ and four tDCS montages commonly used in clinical trials. For the sake of comparison EF components were normalized by the peak value obtained for each electrode montage. Peak values per component, hemisphere, and electrode montage are shown in Table 3 and will be discussed in the next subsection.

Figures 6 and 7 display the coexistence of a tangential component widely spread over the gyri and a normal component mainly focalized in deeper sulci, as has previously been highlighted in the literature [41]. The low thickness and high conductivity of the CSF may partly explain this dual distribution: when the electric current reaches the CSF on its way between the two electrodes through the head, the ions are driven through this thin conductive layer. This occurs tangentially over the thinnest areas (gyri) and perpendicularly in the case of the deepest areas (sulci), which leads to this particularly interesting form of tangential and normal EF distribution over the cortex. It is also noteworthy that unlike the results derived from simplified multilayer spheres mimicking the human head, which show a tangential EF component largely distributed between the electrodes and a normal EF component strongly confined underneath them $[45,46]$, the irregular anatomy of the highly convoluted brain leads to tangential and normal EFs hotspots being dispersed over the cortex [13].

In addition to anatomy, the relative position of the electrodes and the interelectrode distance also constitute key variables which determine the EF distribution 


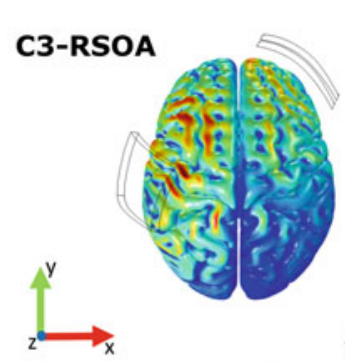

\section{Tangential EF}

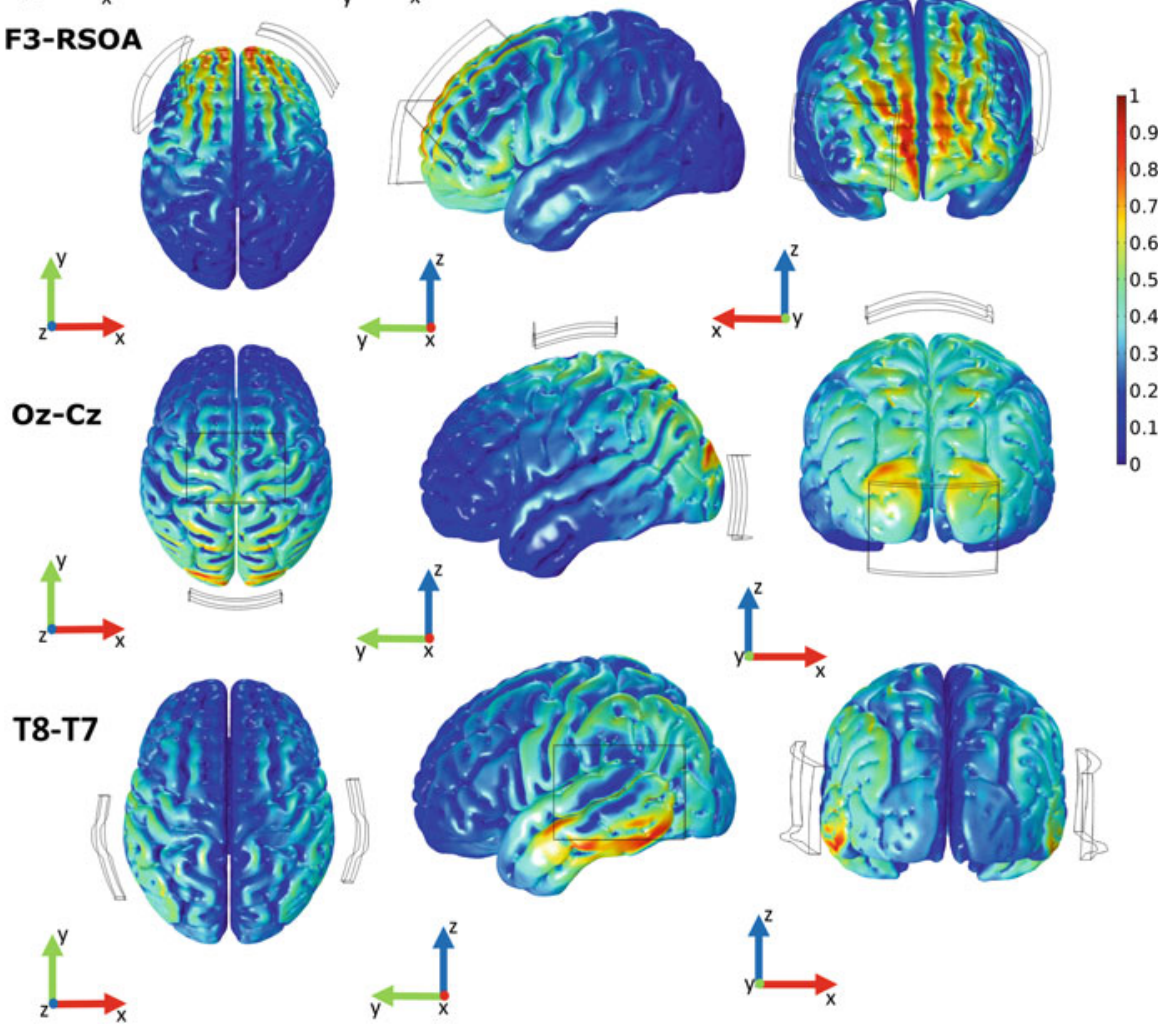

Fig. 6 Normalized magnitude of the tangential EF component for four electrode montages commonly used in clinical applications: C3-RSOA (first row), F3-RSOA (second row), Oz-Cz (third row), and T8-T7 (fourth row)

through the cortical surface [24, 47-51]. For instance, Figs. 6 and 7 show that C3-RSOA exhibits a largely distributed tangential component through the cortex over the whole left hemisphere and the right frontal cortex. This is due to the position of anode C3 in the middle of the left hemisphere, which causes a wider spread of the current lines through the head tissues toward the cathode at RSOA. On the other hand, for the second montage analyzed, F3-RSOA, reducing the inter-electrode 

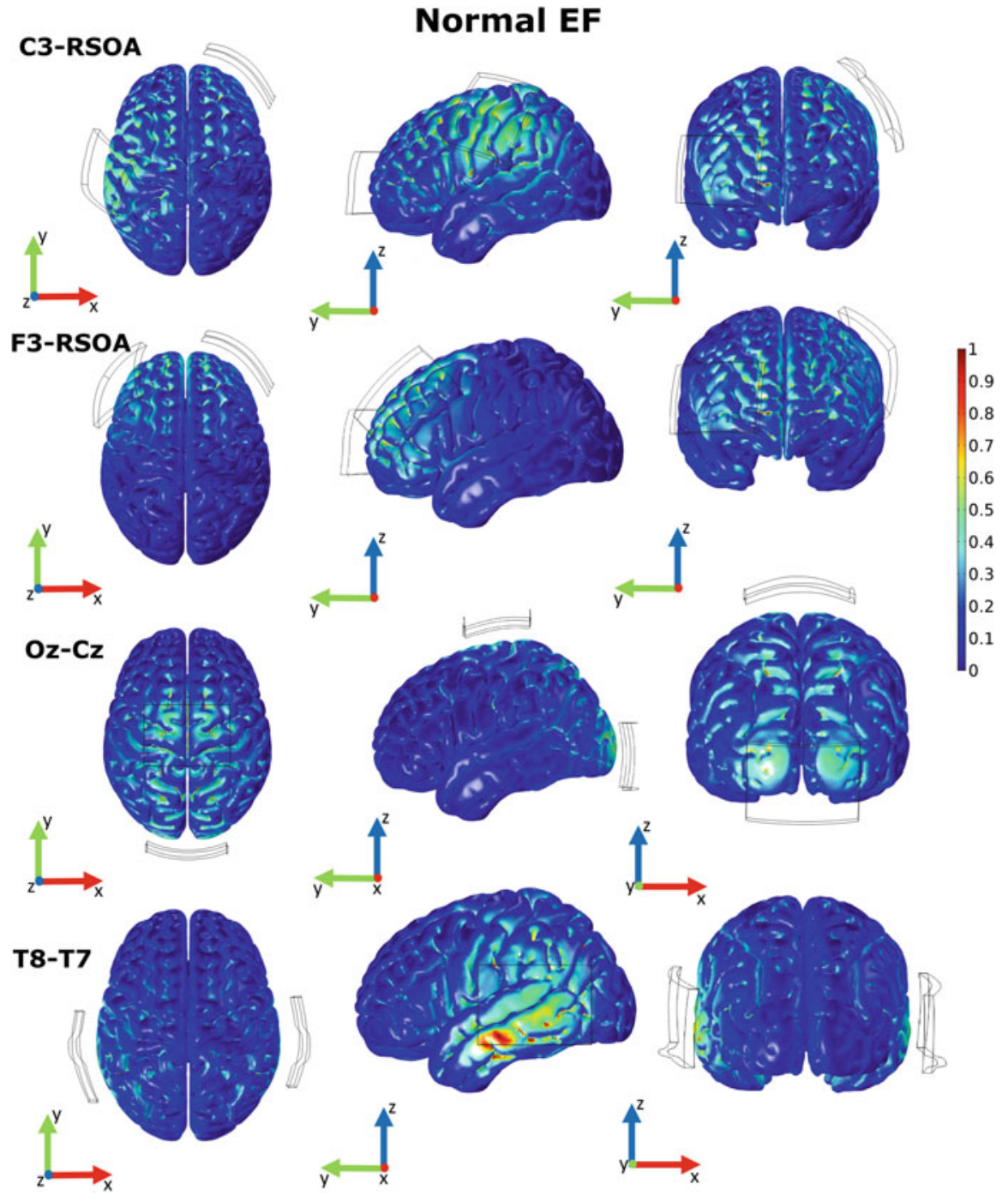

Fig. 7 Normalized magnitude of the normal EF component for four electrode montages commonly used in clinical applications: C3-RSOA (first row), F3-RSOA (second row), Oz-Cz (third row), and T8-T7 (fourth row)

distance by moving the anode from $\mathrm{C} 3$ to $\mathrm{F} 3$ enhances the focality of both tangential and normal $\mathrm{EF}$ in the frontal cortex between the electrodes. In the case of $\mathrm{Oz}-\mathrm{Cz}$, tangential and normal EF are more symmetrically distributed on both hemispheres, mainly over the parietal and occipital cortex, due to the alignment of both the anode and cathode on the midsagittal plane. Finally, T8-T7, with the anode and cathode placed opposite each other, yields an EF pattern mostly confined over the temporal cortex underneath the electrodes. 
Table 3 Peak tangential and normal EF values

\begin{tabular}{l|l|l|l|l}
\hline Peak EF $(\mathrm{V} / \mathrm{m})$ & \multicolumn{4}{l}{} \\
\hline & \multicolumn{2}{l}{ Left hemisphere } & \multicolumn{2}{l}{ Right hemisphere } \\
\cline { 2 - 5 } & $|\overrightarrow{\vec{E}}|$ & $|n \vec{E}|$ & $|t \vec{E}|$ & $|n \vec{E}|$ \\
\hline C3-RSOA & 0.25 & 0.39 & 0.18 & 0.27 \\
\hline F3-RSOA & 0.20 & 0.28 & 0.21 & 0.28 \\
\hline Oz-Cz & 0.23 & 0.29 & 0.21 & 0.29 \\
\hline T8-T7 & 0.29 & 0.31 & 0.19 & 0.31 \\
\hline
\end{tabular}

\subsection{Mean and Peak Tangential and Normal EF Values over Different Cortical Areas}

As shown in Fig. 8, mean tangential $|t \vec{E}|$ and normal $|n \vec{E}|$ EF values were calculated over the 22 cortical areas per hemisphere for 4 electrode montages: C3-RSOA, F3-RSOA, Oz-Cz, and T8-T7. In the majority of cortical areas, the mean $|t \vec{E}|$ values were slightly higher than the mean $|n \vec{E}|$. The peak values over the cortex for the four electrode montages considered are listed in Table 3 and are comparatively shown in Fig. 9. It should also be noted that in the case of $|t \vec{E}|$ peak values were generally twice those of the mean, whereas for $|n \vec{E}|$ this ratio was approximately fourfold. Nevertheless, a number of hemisphere and electrode montage-specific characteristics were also found.

For instance, as expected, some noticeable differences were encountered for C3-RSOA over the right and the left hemispheres, due to its nonsymmetric configuration. As Fig. 8 shows, higher mean $|t \vec{E}|$ and $|n \vec{E}|$ values above $0.10 \mathrm{~V} / \mathrm{m}$ were calculated in regions of the left hemisphere such as SMC, POC, and DLPFC. The highest value of $0.14 \mathrm{~V} / \mathrm{m}$ was obtained on the left IPC near the anode for both $|t \vec{E}|$ and $|n \vec{E}|$. In contrast, lower mean $|t \vec{E}|$ and $|n \vec{E}|$ values were generally found in the majority of areas of the right hemisphere, which is explained by thicker skull and air-filled sinuses under the RSOA electrode. As shown in Fig. 9, peak $|t \vec{E}|$ values of 0.25 and $0.18 \mathrm{~V} / \mathrm{m}$ were estimated over the left IPC and right OPFC, respectively, close to the anode and cathode in each hemisphere. Regarding the normal component, a peak value of about $0.4 \mathrm{~V} / \mathrm{m}$ was predicted on POC just below the bottom edge of the anode on the left hemisphere. Also, a peak value of $0.27 \mathrm{~V} / \mathrm{m}$ was found for $|n \vec{E}|$ over OPFC near the electrode in the right hemisphere. Peak values of 0.2 and $0.25 \mathrm{~V} / \mathrm{m}$ were, calculated in areas such as SMC and PMC.

Compared with C3-RSOA, F3-RSOA resulted in lower mean $|t \vec{E}|$ and $|n \vec{E}|$ EF values below $0.06 \mathrm{~V} / \mathrm{m}$ in the majority of cortical areas on both hemispheres. Higher mean values close to $0.08 \mathrm{~V} / \mathrm{m}$ were only found in areas of the frontal cortex. The enhanced focality, albeit with a lower magnitude, exhibited by this montage is explained by the smaller interelectrode distance. Peak values of about 0.20 and $0.28 \mathrm{~V} / \mathrm{m}$ were, respectively, calculated for $|t \vec{E}|$ and $|n \vec{E}|$ on both right and left 

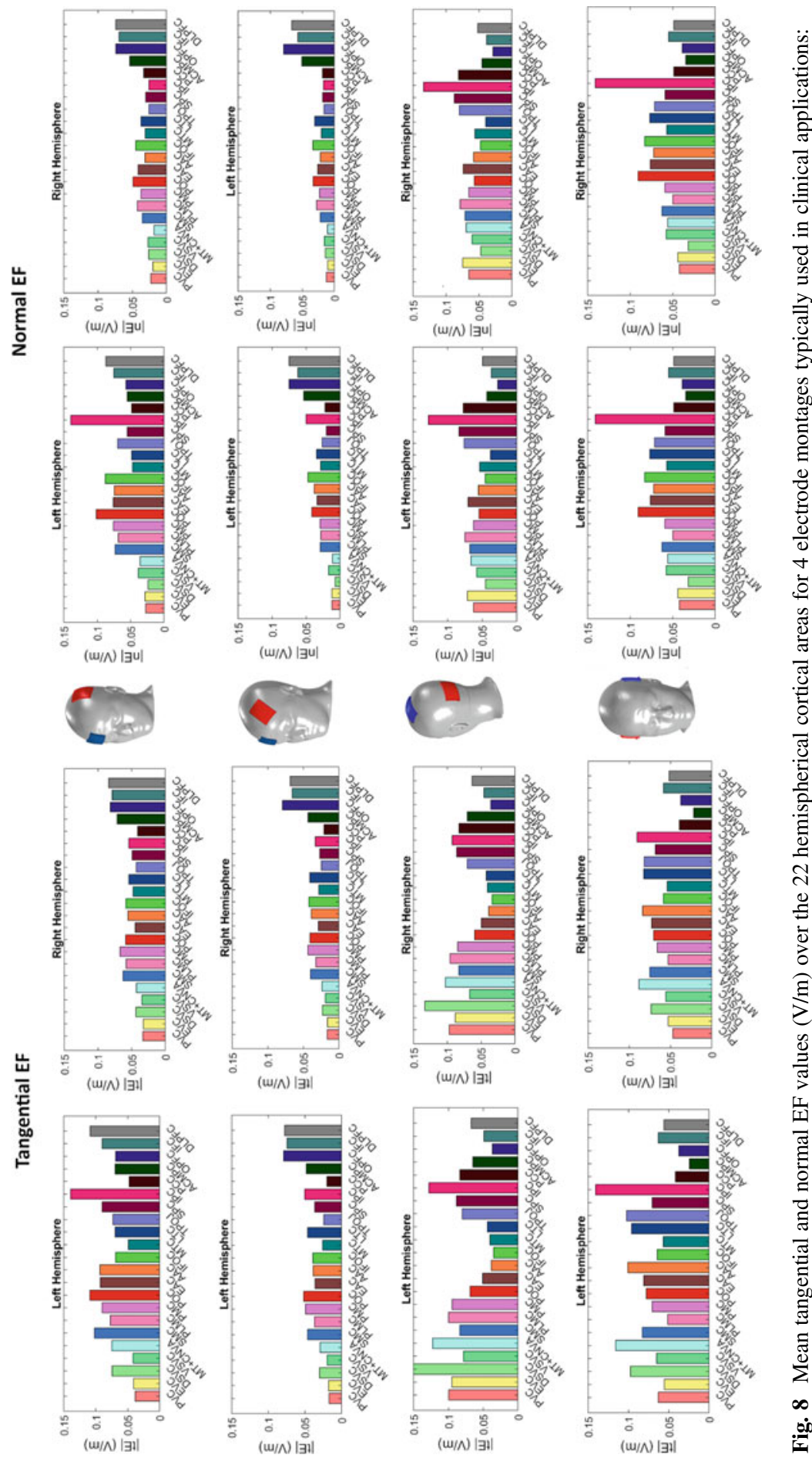

苛
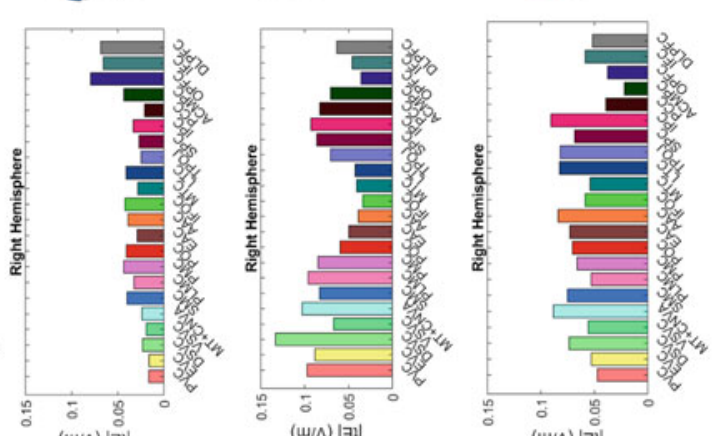

苞

․․

党 $\mathrm{g}$

ฮี

ก

$\underset{ \pm}{ \pm}$

षे

ठ ठ

홍

岂

雪

岌它
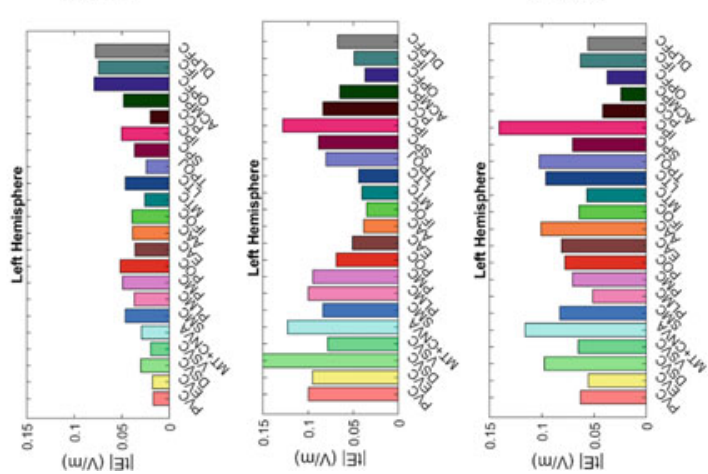

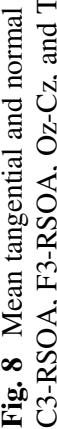



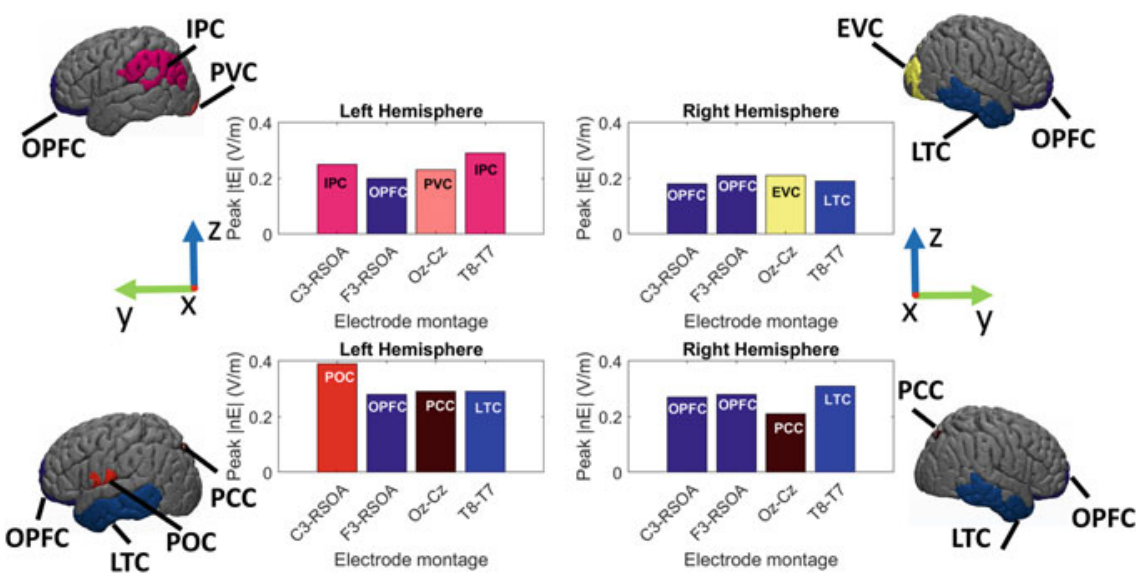

Fig. 9 Simulated peak tangential and normal EF values for the different electrode montages analyzed. The figure also depicts the cortical region where the peak was observed for both right and left hemispheres

OPFC. In addition, a peak normal EF close to $0.28 \mathrm{~V} / \mathrm{m}$ was estimated on the targeted left DLPFC.

In the case of the Oz-Cz montage, higher mean $|\overrightarrow{t E}|$ values of 0.15 and $0.13 \mathrm{~V} / \mathrm{m}$ were calculated for left DSVC and left IPC, respectively, near the superior edge of the anode (Oz). With respect to mean $|n \vec{E}|$ values, slightly lower values below $0.10 \mathrm{~V} / \mathrm{m}$ were found in the majority of cortical areas, except on left and right IPC where a higher mean value near $0.14 \mathrm{~V} / \mathrm{m}$ was seen. Peak $|t \vec{E}|$ values slightly above $0.20 \mathrm{~V} / \mathrm{m}$ were calculated in areas of the visual cortex such as left PVC and right EVC. Interestingly, peak $|n \vec{E}|$ values above $0.20 \mathrm{~V} / \mathrm{m}$ were seen in both left and right PCC, approximately halfway between anode and cathode.

Lastly, mean $|t \vec{E}|$ and $|n \vec{E}|$ values below $0.1 \mathrm{~V} / \mathrm{m}$ were found in the majority of cortical areas for T8-T7. Due to its particular configuration with both electrodes placed above opposite ears, a higher mean value of $0.15 \mathrm{~V} / \mathrm{m}$ was estimated over the left and right IPC near the stimulation electrodes. In the left hemisphere, peak $|\overrightarrow{t E}|$ and $|n \vec{E}|$ values of about $0.3 \mathrm{~V} / \mathrm{m}$ were found in left IPC and LTC, respectively. A high peak value of $0.3 \mathrm{~V} / \mathrm{m}$ was also estimated for $|n \vec{E}|$ on the right LTC just below the anode. 


\section{Summary and Discussion}

A general trend has been confirmed for all the electrode montages analyzed, with a marked anatomical distribution of tangential and normal EF on cortical gyri and sulci, respectively. High mean $|t \vec{E}|$ values were estimated for the majority of cortical regions between the electrodes, which may explain the low spatial resolution often reported for standard tDCS. Instead, higher peak EF values were predicted for $|n \vec{E}|$ in sulci regions underneath and close to the electrode edges, with some electrode montage-specific characteristics.

Specifically, C3-RSOA showed the highest peak values for $|n \vec{E}|$ in areas near the somatosensory and motor cortex and the parietal cortex. This high $|n \vec{E}|$ value in areas below the anode $\mathrm{C} 3$ concurs with recent works that have stressed the relevance of the normal EF component over specific motor targets [20, 29]. However, it must also be noticed that other experimental studies have in fact found a greater correlation between the EF magnitude and the neurophysiological excitatory response on gyral regions such as the left precentral gyrus. For the normal component, a larger intersubject variability prevented the correlation with physiological parameters [31]. In line with these results, our results also show that the EF magnitude in gyri areas mainly accounts for the tangential EF and that a consistent higher-mean gyral tangential EF distribution is observed in the average brain modelled.

The F3-RSOA montage, which targets the left DLPC, exhibited lower $|t \vec{E}|$ and $|n \vec{E}|$ values, possibly due to the presence of air-filled sinuses and thicker skull underneath the electrodes. A more focal EF pattern confined over frontal areas between the electrodes was observed for this montage and is explained by their shorter inter-electrode distance. Peak $|t \vec{E}|$ and $|n \vec{E}|$ values were found over OPFC. These results may corroborate previous modelling studies that have suggested the medial prefrontal cortex as a new target for depression condition in tDCS [10]. Furthermore, previous modelling works analyzing intersubject variability have also reported a greater divergence of EF values over the frontal cortex among subjects for F3-RSOA compared with classic C3-RSOA montage targeting the left motor cortex [6].

Regarding Oz-Cz, this montage showed higher mean and peak $|t \vec{E}|$ values in areas of the occipital/visual cortex just underneath the cathode. Interestingly, peak $|n \vec{E}|$ values were also seen on PCC halfway between both stimulation electrodes, thus corroborating prior modelling results in [12].

In the case of T7-T8, previous computational results on auditory tDCS reported higher mean values of current density confined around the stimulation electrodes [52]. Our results here coincide with these previous outcomes showing both hemispheres being stimulated with higher mean and peak $|t \vec{E}|$ and $|n \vec{E}|$ EF values over regions of the auditory cortex and LTC near the stimulation electrodes. Finally, it is noteworthy that three of the four montages analyzed, C3-RSOA, Oz-Cz, and T8-T7, 
showed higher mean tangential and normal EF values over IPC, possibly explained by local CSF thinning.

\section{Conclusion}

In this chapter we have conducted a computational analysis of the distribution of the tangential and normal components of the electric field over a representative parcellated brain-FEM model for different electrode montages commonly used in tDCS clinical applications. The results confirmed the existence of a dual EF pattern on the cortex, based on a widely gyri-distributed tangential component and a sulciconfined normal EF component. One open question is if such a noticeable dependence of EF components on cortical morphology is the cause of the low focality reported for large-pad tDCS or if, conversely, it actually entails significant directionbased mechanisms for the stimulation outcome at distinct neural segments. Therefore, the validation of computational EF models with physiological measurements is vital in order to clarify this controversy. Our analysis could prove helpful in designing new experimental studies which allow greater understanding of the underlying modulatory mechanisms related to different EF components in tDCS.

\section{References}

1. Stagg, C. J., Antal, A., \& Nitsche, M. A. (2018, September). Physiology of transcranial direct current stimulation. The Journal of ECT, 34(3), 144-152.

2. Truong, D. Q., \& Bikson, M. (2018, September). Physics of transcranial direct current stimulation devices and their history. The Journal of ECT, 34(3), 137-143.

3. Morya, E., et al. (2019, November). Beyond the target area: An integrative view of tDCSinduced motor cortex modulation in patients and athletes. Journal of Neuroengineering and Rehabilitation, 16(1), 1-29.

4. Yavari, F., Jamil, A., Mosayebi Samani, M., Vidor, L. P., \& Nitsche, M. A. (2018, February). Basic and functional effects of transcranial electrical stimulation (tES) - An introduction. Neuroscience and Biobehavioral Reviews, 85, 81-92.

5. Bikson, M., et al. (2018, May). Rigor and reproducibility in research with transcranial electrical stimulation: An NIMH-sponsored workshop. Brain Stimulation, 11(3), 465-480.

6. Laakso, I., Tanaka, S., Mikkonen, M., Koyama, S., Sadato, N., \& Hirata, A. (2016, August). Electric fields of motor and frontal tDCS in a standard brain space: A computer simulation study. NeuroImage, 137, 140-151.

7. Ciechanski, P., Carlson, H. L., Yu, S. S., \& Kirton, A. (2018, July). Modeling transcranial direct-current stimulation-induced electric fields in children and adults. Frontiers in Human Neuroscience, 12, 1-14.

8. Mikkonen, M., Laakso, I., Tanaka, S., \& Hirata, A. (2020, January). Cost of focality in TDCS: Interindividual variability in electric fields. Brain Stimulation, 13(1), 117-124.

9. Evans, C., Bachmann, C., Lee, J. S. A., Gregoriou, E., Ward, N., \& Bestmann, S. (2020, January). Dose-controlled tDCS reduces electric field intensity variability at a cortical target site. Brain Stimulation, 13(1), 125-136. 
10. Csifcsák, G., Boayue, N. M., Puonti, O., Thielscher, A., \& Mittner, M. (2018, July). Effects of transcranial direct current stimulation for treating depression: A modeling study. Journal of Affective Disorders, 234, 164-173.

11. Huang, Y., Dmochowski, J. P., Su, Y., Datta, A., Rorden, C., \& Parra, L. C. (2013, December). Automated MRI segmentation for individualized modeling of current flow in the human head. Journal of Neural Engineering, 10(6), 1-26.

12. Rampersad, S. M., et al. (2014, May). Simulating transcranial direct current stimulation with a detailed anisotropic human head model. IEEE Transactions on Neural Systems and Rehabilitation Engineering, 22(3), 441-452.

13. Opitz, A., Paulus, W., Will, S., Antunes, A., \& Thielscher, A. (2015, April). Determinants of the electric field during transcranial direct current stimulation. NeuroImage, 109, 140-150.

14. Gomez-Tames, J., Asai, A., \& Hirata, A. (2019, December). Significant group-level hotspots found in deep brain regions during transcranial direct current stimulation (tDCS): A computational analysis of electric fields. Clinical Neurophysiology, 131, 755-765.

15. Bestmann, S., \& Ward, N. (2017, July). Are current flow models for transcranial electrical stimulation fit for purpose? Brain Stimulation, 10(4), 865-866.

16. Peterchev, A. V. (2017, March). Transcranial electric stimulation seen from within the brain. eLife, 6, 1-3.

17. Polanía, R., Nitsche, M. A., \& Ruff, C. C. (2018, February). Studying and modifying brain function with non-invasive brain stimulation. Nature Neuroscience, 21(2), 174-187.

18. Rawji, V., et al. (2018, March). tDCS changes in motor excitability are specific to orientation of current flow. Brain Stimulation Basic Translation and Clinical Research in Neuromodulation, 11(2), 289-298.

19. Hannah, R., Iacovou, A., \& Rothwell, J. C. (2019, May). Direction of TDCS current flow in human sensorimotor cortex influences behavioural learning. Brain Stimulation, 12(3), 684-692.

20. Laakso, I., Mikkonen, M., Koyama, S., Hirata, A., \& Tanaka, S. (2019, December). Can electric fields explain inter-individual variability in transcranial direct current stimulation of the motor cortex? Scientific Reports, 9(1), 1-10.

21. Callejon-Leblic, M. A., \& Miranda, P. C. (2019). A computational analysis of the electric field components in transcranial direct current stimulation. In: 41st Annual International Conference of the IEEE Engineering in Medicine and Biology Society (EMBC), Berlin, Germany, pp. 5913-5917, July 2019.

22. Nitsche, M. A., \& Paulus, W. (2000, September). Excitability changes induced in the human motor cortex by weak transcranial direct current stimulation. The Journal of Physiology, 527(3), 633-639.

23. Nitsche, M. A., et al. (2007, April). Shaping the effects of transcranial direct current stimulation of the human motor cortex. Journal of Neurophysiology, 97(4), 3109-3117.

24. Opitz, A., Yeagle, E., Thielscher, A., Schroeder, C., Mehta, A. D., \& Milham, M. P. (2018, November). On the importance of precise electrode placement for targeted transcranial electric stimulation. NeuroImage, 181, 560-567.

25. Huang, Y., \& Parra, L. C. (2019, January). Can transcranial electric stimulation with multiple electrodes reach deep targets? Brain Stimulation, 12(1), 30-40.

26. Liu, A., et al. (2018, vs). Immediate neurophysiological effects of transcranial electrical stimulation. Nature Communications, 9(1), 1-10.

27. Chakraborty, D., Truong, D. Q., Bikson, M., \& Kaphzan, H. (2018, August). Neuromodulation of axon terminals. Cerebral Cortex, 28(8), 2786-2794.

28. Rahman, A., et al. (2013, May). Cellular effects of acute direct current stimulation: Somatic and synaptic terminal effects. The Journal of Physiology, 591(10), 2563-2578.

29. Seo, H., \& Jun, S. C. (2019, March). Relation between the electric field and activation of cortical neurons in transcranial electrical stimulation. Brain Stimulation, 12(2), 275-289.

30. Fischer, D. B., et al. (2017, August). Multifocal tDCS targeting the resting state motor network increases cortical excitability beyond traditional tDCS targeting unilateral motor cortex. NeuroImage, 157, 34-44. 
31. Antonenko, D., et al. (2019, September). Towards precise brain stimulation: Is electric field simulation related to neuromodulation? Brain Stimulation, 12(5), 1159-1168.

32. Foerster, Á., et al. (2019, March). Effects of electrode angle-orientation on the impact of transcranial direct current stimulation on motor cortex excitability. Brain Stimulation, 12(2), 263-266.

33. Miranda, P. C., Callejón-Leblic, M. A., Salvador, R., \& Ruffini, G. (2018, December). Realistic modeling of transcranial current stimulation: The electric field in the brain. Current Opinion Biomedical Engineering, 8, 20-27.

34. Fonov, V., Evans, A. C., Botteron, K., Almli, C. R., McKinstry, R. C., \& Collins, D. L. (2011, January). Unbiased average age-appropriate atlases for pediatric studies. NeuroImage, 54(1), 313-327.

35. Windhoff, M., Opitz, A., \& Thielscher, A. (2013, April). Electric field calculations in brain stimulation based on finite elements: An optimized processing pipeline for the generation and usage of accurate individual head models. Human Brain Mapping, 34(4), 923-935.

36. Nielsen, J. D., et al. (2018, July). Automatic skull segmentation from MR images for realistic volume conductor models of the head: Assessment of the state-of-the-art. NeuroImage, 174, 587-598.

37. Huang, Y., Parra, L. C., \& Haufe, S. (2016, October). The New York Head-A precise standardized volume conductor model for EEG source localization and tES targeting. NeuroImage, 140, 150-162.

38. Eickhoff, S. B., Yeo, B. T. T., \& Genon, S. (2018, November). Imaging-based parcellations of the human brain. Nature Reviews Neuroscience, 19(11), 672-686.

39. Glasser, M. F., et al. (2016, August). A multi-modal parcellation of human cerebral cortex. Nature, 536(7615), 171-178.

40. Mills, K. (2016). HCP-MMP1.0 projected on fsaverage. FigShare (dataset). https://doi.org/10. 6084/m9.figshare.3498446.v2. Last access: December 2019.

41. Miranda, P. C., Mekonnen, A., Salvador, R., \& Ruffini, G. (2013, April). The electric field in the cortex during transcranial current stimulation. NeuroImage, 70, 48-58.

42. Makarov, S. N., Noetscher, G. M., \& Nazarian, A. (2016). Low-frequency electromagnetic modeling for electrical and biological systems using MATLAB. Hoboken: Wiley., ISBN: 978-1119-05256-2.

43. Makarov, S. N., et al. (2017, June). Virtual human models for electromagnetic studies and their applications. IEEE Reviews in Biomedical Engineering, 10, 95-121.

44. Koessler, L., et al. (2017, February). In-vivo measurements of human brain tissue conductivity using focal electrical current injection through intracerebral multicontact electrodes. Human Brain Mapping, 38(2), 974-986.

45. Faria, P., Hallett, M., \& Miranda, P. C. (2011 December). A finite element analysis of the effect of electrode area and inter-electrode distance on the spatial distribution of the current density in tDCS. Journal of Neural Engineering, 8(6), 066017.

46. Truong, D. Q., et al. (2014, July). Clinician accessible tools for GUI computational models of transcranial electrical stimulation: BONSAI and SPHERES. Brain Stimulation, 7(4), 521-524.

47. Ramaraju, S., Roula, M. A., \& McCarthy, P. W. (2018, February). Modelling the effect of electrode displacement on transcranial direct current stimulation (tDCS). Journal of Neural Engineering, 15(1), 1-7.

48. Saturnino, G. B., Antunes, A., \& Thielscher, A. (2015, October). On the importance of electrode parameters for shaping electric field patterns generated by tDCS. NeuroImage, 120, 25-35.

49. Salvador, R., Wenger, C., Nitsche, M. A., \& Miranda, P. C. (2015, November). How electrode montage affects transcranial direct current stimulation of the human motor cortex. In: Proceedings of the Annual International Conference of the IEEE Engineering in Medicine and Biology Society, EMBS, pp. 6924-6927.

50. Bai, S., Dokos, S., Ho, K. A., \& Loo, C. (2014, February). A computational modelling study of transcranial direct current stimulation montages used in depression. NeuroImage, 87, 332-344. 
51. Bikson, M., Datta, A., Rahman, A., \& Scaturro, J. (2010, December). Electrode montages for tDCS and weak transcranial electrical stimulation: Role of 'return' electrode's position and size. Clinical Neurophysiology, 121(12), 1976-1978.

52. Wagner, S., et al. (2014, February). Investigation of tDCS volume conduction effects in a highly realistic head model. Journal of Neural Engineering, 11(1), 1-14.

Open Access This chapter is licensed under the terms of the Creative Commons Attribution 4.0 International License (http://creativecommons.org/licenses/by/4.0/), which permits use, sharing, adaptation, distribution and reproduction in any medium or format, as long as you give appropriate credit to the original author(s) and the source, provide a link to the Creative Commons license and indicate if changes were made.

The images or other third party material in this chapter are included in the chapter's Creative Commons license, unless indicated otherwise in a credit line to the material. If material is not included in the chapter's Creative Commons license and your intended use is not permitted by statutory regulation or exceeds the permitted use, you will need to obtain permission directly from the copyright holder.

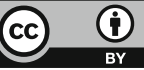

